\title{
Early Impact of the COVID-19 Pandemic on Acute Stroke Treatment Delays
}

\author{
Joel Neves Briard (i), Célina Ducroux, Grégory Jacquin (1D, Walid Alesefir, \\ William Boisseau, Nicole Daneault, Yan Deschaintre, Johanna Eneling, \\ Laura C. Gioia (1), Dana Iancu, Céline Odier, Jean Raymond, Daniel Roy, \\ Christian Stapf, Alain Weill, Alexandre Y. Poppe (D)
}

\begin{abstract}
This is an observational cohort study comparing 156 patients evaluated for acute stroke between March 30 and May 31 , 2020 at a comprehensive stroke center with 138 patients evaluated during the corresponding time period in 2019. During the pandemic, the proportion of COVID-19 positive patients was low (3\%), the time from symptom onset to hospital presentation was significantly longer, and a smaller proportion of patients underwent reperfusion therapy. Among patients directly evaluated at our institution, door-toneedle and door-to-recanalization metrics were significantly longer. Our findings support concerns that the current pandemic may have a negative impact on the management of acute stroke.
\end{abstract}

RÉSUMÉ : Les premiers effets de la pandémie de COVID-19 en matière de délais dans le traitement d'AVC aigus. Il s'agit d'une étude de cohorte observationnelle ayant comparé 156 patients évalués dans un centre de soins complets de l'AVC à la suite d'un AVC aigu survenu du 30 mars au 31 mai 2020 à 138 patients qui avaient été évalués durant la même période en 2019. Au cours de cette pandémie, il est vrai que la proportion de patients ayant testé positifs à la COVID-19 est demeurée faible (3\%). Cela dit, les délais entre l'apparition des symptômes d'un AVC et la présentation dans un établissement hospitalier se sont révélés notablement plus longs. De plus, une proportion moins élevée de patients ont pu bénéficier d'une thérapie de reperfusion. Parmi les patients évalués directement dans notre établissement, les délais entre l'arrivée à l'hôpital et l'injection d'un traitement (door-to-needle), de même que les délais entre l'arrivée à l'hôpital et une procédure de recanalisation artérielle, se sont allongés de façon importante. En somme, nos résultats entérinent les préoccupations selon lesquelles la pandémie actuelle pourrait avoir un impact négatif sur la prise en charge de patients victimes d'un AVC aigu.

Keywords: Acute stroke, Coronavirus, COVID-19, SARS-CoV-2, Comprehensive stroke center doi: $10.1017 /$ cjn. 2020.160

Can J Neurol Sci. 2021; 48: 122-126

With the outbreak of coronavirus disease 2019 (COVID-19), usual stroke care logistics have been disrupted worldwide. ${ }^{1}$ Guidelines currently recommend screening suspected stroke patients for COVID-19 and employing appropriate precautions to prevent contamination of healthcare workers and other patients, ${ }^{2,3}$ while some groups have proposed protected acute stroke evaluation algorithms. ${ }^{4}$ Our institution's modified code stroke protocol includes use of personal protective equipment during all acute stroke evaluations and systematic COVID-19 screening for all code stroke patients with a nasopharyngeal swab and polymerase chain reaction (PCR). We aimed to study the impact of the COVID-19 pandemic on stroke treatment metrics.

We conducted an observational cohort study at a high-volume comprehensive stroke center for which exposure was defined as the emerging COVID-19 pandemic. Montreal, Quebec, is currently the epicenter of the COVID-19 pandemic in Canada $(21,912$ confirmed cases as of May 31, 2020). Using data from the Montreal Neurovascular and Stroke Data Repository (MONSTER), a routine electronic medical record tool allowing systematic guideline-based quality assurance, we compared a prospective cohort of consecutive adult patients evaluated for acute stroke between March 30 (date of formal implementation of our modified code stroke protocol) and May 31, 2020, with a retrospective reference cohort of consecutive adult patients evaluated for stroke during the corresponding time period of 2019. We collected baseline characteristics, prestroke modified Rankin scale (mRS) and NIH stroke scale (NIHSS) scores, discharge diagnosis (stratified into ischemic stroke, hemorrhagic stroke, stroke mimic), reperfusion treatment (thrombolysis, thrombectomy), time between symptom onset and hospital presentation, stroke management metrics (door-to-imaging, door-toneedle, door-to-puncture, and door-to-recanalization delays), and the COVID-19 screening results. Statistical testing was performed using $t$-tests (continuous parametric data), Wilcoxon rank-sum tests (continuous nonparametric data), and $\chi^{2}$ tests (dichotomous data). For ischemic stroke patients, multivariate logistic regressions were used with the following prespecified variables: stroke evaluation during the pandemic period, patient age, sex, prestroke $\mathrm{mRS}$,

From the Department of Neurosciences, Faculté de médecine, Université de Montréal, Montréal, Canada (JNB, CD, GJ, WA, ND, YD, LCG, CO, CS, AYP); Department of Medicine (Neurology), Centre Hospitalier de l'Université de Montréal, Montréal, Canada (JNB, CD, GJ, WA, ND, YD, LCG, CO, CS, AYP); Neurovascular Group, Neurosciences Axis, Centre de Recherche du Centre Hospitalier de l'Université de Montréal (CRCHUM), Montréal, Canada (GJ, ND, YD, LCG, DI, CO, JR, DR, CS, AW, AYP); and Department of Radiology (Neuroradiology), Centre Hospitalier de l'Université de Montréal, Montréal, Canada (WB, JE, DI, JR, DR, AW)

Received June 20, 2020. Final Revisions Submitted July 2, 2020. Date of ACCEPTANCE July 21, 2020.

Correspondence to: Alexandre Y. Poppe, Department of Medicine (Neurology), Centre Hospitalier del'Université de Montréal, 900 rue St-Denis, Bureau R04-758, Montréal, Québec, Canada H2X 3H8. Email: alexandre.poppe.chum@ssss.gouv.qc.ca 
Table 1: Characteristics of the pandemic and reference cohorts

\begin{tabular}{|c|c|c|c|}
\hline & $\begin{array}{c}2020 \text { pandemic cohort } \\
(n=156)\end{array}$ & $\begin{array}{l}2019 \text { reference cohort } \\
(n=138)\end{array}$ & $p$ \\
\hline \multicolumn{4}{|l|}{ Demographic data } \\
\hline Age, mean (SD) & $69.4(16.1)$ & $72.1(14.9)$ & 0.13 \\
\hline Sex, $N$, male $(\%)$ & $75(48)$ & $71(51)$ & 0.56 \\
\hline \multicolumn{4}{|l|}{ Baseline comorbidities, $N(\%)$} \\
\hline Hypertension & $84(54)$ & $87(63)$ & 0.11 \\
\hline Dyslipidemia & $64(41)$ & $57(41)$ & 0.96 \\
\hline Diabetes mellitus & $38(24)$ & $31(22)$ & 0.70 \\
\hline Atrial fibrillation or flutter & $19(12)$ & $24(17)$ & 0.21 \\
\hline Prior ischemic stroke or transient ischemic attack & $36(23)$ & $29(21)$ & 0.67 \\
\hline Prior intracranial hemorrhage & $4(3)$ & $2(1)$ & 0.50 \\
\hline Coronary or peripheral artery disease & $21(13)$ & $23(17)$ & 0.44 \\
\hline Tobacco use & $26(17)$ & $26(19)$ & 0.63 \\
\hline Excessive alcohol use & $10(6)$ & $13(9)$ & 0.34 \\
\hline \multicolumn{4}{|l|}{ Prior antithrombotic treatment, $N(\%)$} \\
\hline Antiplatelet & $44(28)$ & $44(31)$ & 0.49 \\
\hline Direct anticoagulant & $16(10)$ & $11(8)$ & 0.50 \\
\hline Vitamin $\mathrm{K}$ antagonist & $8(5)$ & $2(1)$ & 0.08 \\
\hline \multicolumn{4}{|l|}{ Patient flow, $N(\%)$} \\
\hline Direct evaluation & $121(78)$ & $90(65)$ & \multirow[t]{2}{*}{0.02} \\
\hline Transfer from another institution & $35(22)$ & $48(35)$ & \\
\hline \multicolumn{4}{|l|}{ ED assessment, median (IQR) } \\
\hline Prestroke mRS & $1(0-2)$ & $1(0-2)$ & 0.95 \\
\hline NIHSS & $9(3-20)$ & $12(5-19)$ & 0.40 \\
\hline \multicolumn{4}{|l|}{ Diagnosis, $N(\%)$} \\
\hline Ischemic stroke & $104(67)$ & $93(68)$ & \multirow[t]{3}{*}{0.35} \\
\hline Hemorrhagic stroke & $17(11)$ & $18(13)$ & \\
\hline Stroke mimic & $35(22)$ & $25(19)$ & \\
\hline \multicolumn{4}{|l|}{ Ischemic stroke treatment, $N(\%)$} \\
\hline Thrombolysis & $38(36)$ & $51(54)$ & 0.01 \\
\hline Thrombectomy & $38(36)$ & $51(54)$ & 0.01 \\
\hline Under general anesthesia & $5(13)$ & $4(8)$ & 0.41 \\
\hline \multicolumn{4}{|c|}{ Delays and ischemic stroke management metrics in minutes, median (IQR) } \\
\hline \multicolumn{4}{|l|}{ Symptom onset to hospital presentation } \\
\hline All patients & $147(64-363)$ & $102(57-205)$ & 0.02 \\
\hline All ischemic stroke patients & $197(64-501)$ & $116(60-212)$ & 0.03 \\
\hline \multicolumn{4}{|l|}{ Door-to-imaging } \\
\hline Direct evaluation & $17(13-22)$ & $16(10-21)$ & 0.16 \\
\hline Transfer from another institution & $29(19-43)$ & $20(13-30)$ & 0.04 \\
\hline \multicolumn{4}{|l|}{ Door-to-needle } \\
\hline Direct evaluation & $34(25-41)$ & $22(21-30)$ & $<0.01$ \\
\hline Transfer from another institution & $59(45-80)$ & $55(44-74)$ & 0.86 \\
\hline \multicolumn{4}{|l|}{ Door-to-puncture } \\
\hline Direct evaluation & $69(61-97)$ & $56(46-73)$ & 0.09 \\
\hline Transfer from another institution(door: referring ED) & $145(134-167)$ & $150(134-167)$ & 0.65 \\
\hline Transfer from another institution(door: our ED) & $21(17-25)$ & $15(11-26)$ & 0.14 \\
\hline
\end{tabular}


Table 1. (Continued)

\begin{tabular}{l|c|c|c}
\hline & $\begin{array}{c}\mathbf{2 0 2 0} \text { pandemic cohort } \\
(\boldsymbol{n}=\mathbf{1 5 6})\end{array}$ & $\begin{array}{c}\mathbf{2 0 1 9} \text { reference cohort } \\
(\boldsymbol{n}=\mathbf{1 3 8})\end{array}$ & $\boldsymbol{p}$ \\
\hline Door-to-recanalization & $100(90-124)$ & $89(64-97)$ & 0.04 \\
\hline Patients directly evaluated at our institution & $190(161-221)$ & $179(135-224)$ & 0.60 \\
\hline Transfer from another institution(door: referring ED) & $51(40-69)$ & $50(35-67)$ & 0.54 \\
\hline Transfer from another institution(door: our ED) &
\end{tabular}

$\mathrm{SD}=$ standard deviation; $\mathrm{IQR}=$ interquartile range; $\mathrm{ED}=$ emergency department.

Table 2: Logistic regressions for acute reperfusion therapies

\begin{tabular}{l|c|c}
\hline & $\begin{array}{c}\text { Thrombolysis } \\
\mathbf{a O R , 9 5 \% ~ C I}\end{array}$ & $\begin{array}{c}\text { Thrombectomy } \\
(\mathbf{a O R}, \mathbf{9 5 \%} \text { CI })\end{array}$ \\
\hline Stroke evaluation during the pandemic period & $0.49(0.23-1.03)$ & $0.70(0.26-1.86)$ \\
\hline Age & $1.01(0.99-1.04)$ & $0.98(0.95-1.02)$ \\
\hline Sex (male) & $1.18(0.57-2.46)$ & $1.04(0.38-2.87)$ \\
\hline $\begin{array}{l}\text { Delay from symptom onset }(<4.5 \mathrm{~h} \text { for thrombolysis, } \\
<6 \text { h for thrombectomy) }\end{array}$ & $4.51(1.94-11.27)$ & $0.80(0.22-2.81)$ \\
\hline Transfer from another institution & $1.04(0.47-2.28)$ & $9.49(3.66-27.19)$ \\
\hline NIHSS & $1.08(1.03-1.13)$ & $1.28(1.19-1.40)$ \\
\hline Prestroke mRS & $0.50(0.33-0.72)$ & $0.66(0.40-0.97)$ \\
\hline
\end{tabular}

$\mathrm{aOR}=$ adjusted odds ratio; $95 \% \mathrm{CI}=95 \%$ confidence interval.

NIHSS, transfer from another institution, and delay from symptom onset. The outcomes of interest were treatment with thrombolysis and thrombectomy, hospital presentation within $4.5 \mathrm{~h}$ from symptom onset, and referral from another institution for thrombectomy. The project was approved by our institutional Research Ethics Board (\#20.013).

Overall, 156 patients were included in the pandemic cohort and 138 in the reference cohort. Baseline characteristics and stroke subtypes were similar between cohorts (Table 1; see Supplementary Table for data on confirmed ischemic stroke patients). Delays to hospital presentation were longer during the pandemic for all evaluated patients (median [interquartile range]: 147 [64-363] vs. 102 [57-205] $\mathrm{min}, p=0.02$ ) and for ischemic stroke patients (197 [64-501] vs. 116 [60-212] min, $p=0.03$ ). During the pandemic, a significantly smaller proportion of ischemic stroke patients was treated with thrombolysis or thrombectomy (36\% vs. $54 \%$ for both interventions, $p=0.01$ ) and fewer patients were transferred from another institution for thrombectomy (22\% vs. $35 \%, p=0.02$ ). Furthermore, patients directly evaluated at our institution had longer door-to-needle (34 [25-41] vs. 22 [21-30] $\min , p<0.01)$ and door-to-recanalization delays (100 [90-124] vs. 89 [64-97] $\mathrm{min}, p=0.04$ ).

The proportion of COVID-19 positive cases was 3\% (4/146); two patients presented with stroke mimics (septic encephalopathy) and two had confirmed ischemic strokes. Ten patients, all diagnosed as stroke mimics, had declined screening.

When controlling for confounders, the pandemic period was not statistically associated with thrombolysis treatment (adjusted odds ratio [aOR]: 0.49, 95\% confidence interval [CI]: 0.23-1.03) or thrombectomy (aOR: 0.70, 95\% CI: 0.26-1.86; Table 2).
However, the pandemic period was inversely associated with hospital presentation within $4.5 \mathrm{~h}$ from symptom onset (aOR: 0.46, 95\% CI: 0.21-0.96) and with referral from another institution for thrombectomy (aOR: 0.40, 95\% CI: 0.19-0.82; Table 3).

This study provides additional evidence that the COVID-19 pandemic has an impact on the presentation and management of acute stroke patients. While in some areas stroke centers have reported a lower number of acute stroke evaluations overall, ${ }^{5-8}$ we observed a similar acute stroke caseload during the first 2 months of the pandemic. This may reflect increased referrals to our center resulting from temporary closures of nearby stroke centers due to institutional outbreaks during the study period. Importantly, delays between symptom onset and hospital presentation were significantly longer, and fewer patients underwent reperfusion therapy. Our analysis suggests that the pandemic may be affecting reperfusion therapy eligibility through increased delays to hospital presentation and decreased referrals from other institutions.

In addition, we observed longer delays to reperfusion (doorto-needle and door-to-recanalization) and only $3 \%$ of patients testing positive for COVID-19 (Figure 1). Albeit necessary, in-hospital infection control measures, including the use of appropriate protective equipment and secure patient transportation, may be delaying acute stroke management. Other than these safety procedures, we avoided modifications to our established, guideline-based code stroke protocol. For instance, patient transfer from first evaluation to CT scan remained streamlined with prenotification and dedicated transport staff, which may explain why we did not observe an increase in door-to-imaging delays. While some authors have advocated greater use of elective 
Table 3: Logistic regressions for hospital presentation within $4.5 \mathrm{~h}$ from symptom onset and referral from another institution for endovascular procedures

\begin{tabular}{l|c|c}
\hline & $\begin{array}{c}\text { Hospital presentation within 4.5 h } \\
(\mathbf{a O R}, 95 \% \text { CI })\end{array}$ & $\begin{array}{c}\text { Referral from another institution } \\
\text { (aOR, 95\% CI) }\end{array}$ \\
\hline Stroke evaluation during the pandemic period & $0.46(0.21-0.96)$ & $0.40(0.19-0.82)$ \\
\hline Age & $1.00(0.97-1.02)$ & $0.98(0.96-1.01)$ \\
\hline Sex (male) & $1.01(0.49-2.08)$ & $1.39(0.67-2.93)$ \\
\hline Delay from symptom onset & N/A & $0.64(0.25-1.64)$ \\
\hline Transfer from another institution & $1.23(0.54-2.89)$ & $\mathrm{N} / \mathrm{A}$ \\
\hline NIHSS & $1.01(0.97-1.06)$ & $1.11(1.06-1.16)$ \\
\hline Prestroke mRS & $0.75(0.54-0.99)$ & $0.63(0.44-0.89)$ \\
\hline
\end{tabular}

$\mathrm{aOR}=$ adjusted odds ratio; $95 \% \mathrm{CI}=95 \%$ confidence interval.
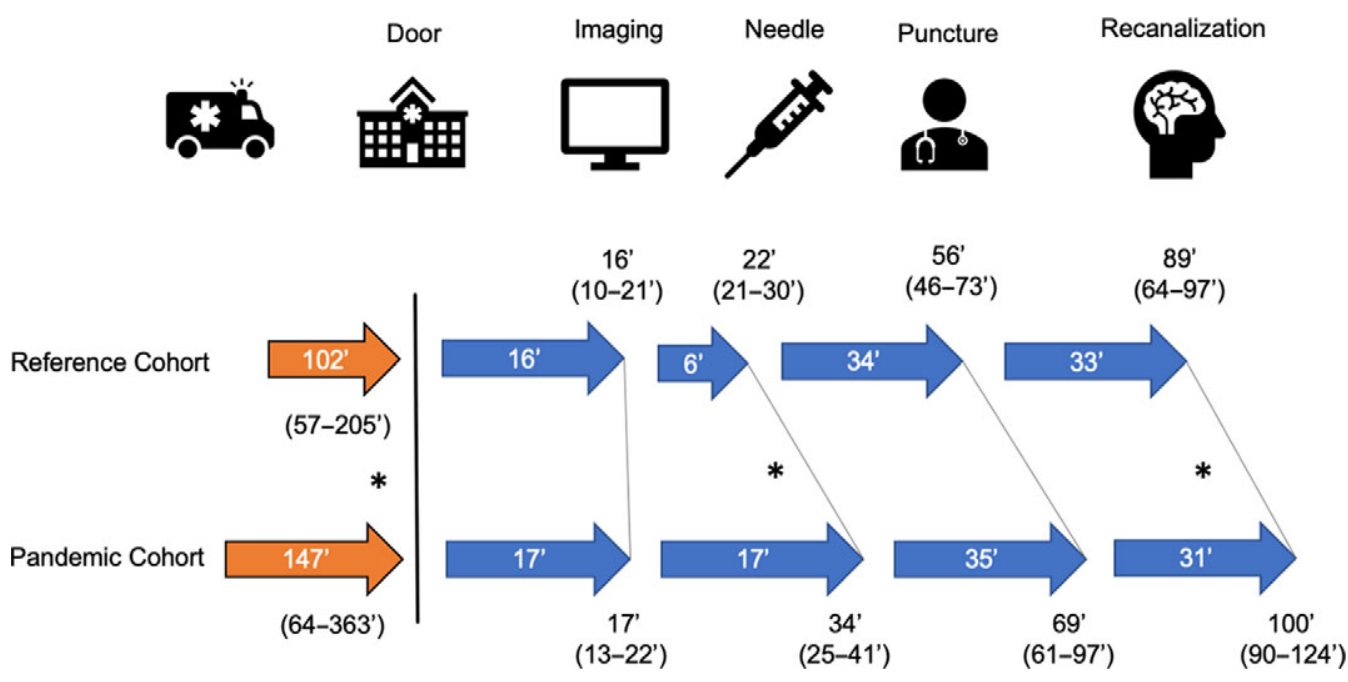

Figure 1: Comparative stroke metrics. Data presented as medians (interquartile ranges). *Statistically significant differences.

intubation for endovascular therapy during the pandemic, ${ }^{9}$ our approach remained unchanged in order to avoid further delays. Existence of an established code stroke protocol with prior favorable metrics, as well as collaborative implementation of required protocol modifications, may explain why despite greater delays our in-house metrics still remained within the accepted range of recommended clinical standards during the pandemic.

Our study is limited by its single-center design and by the relatively short observation period. Although demographics and clinical characteristics were similar between cohorts, the possibility of systematic or random bias cannot be excluded. Whether the longer delays observed in this study will be sustained over time and translate into worse functional outcomes for patients remains to be determined and will be evaluated in longer term studies.

In conclusion, the first two months of the COVID-19 pandemic were not associated with a decrease in acute stroke evaluations in our comprehensive stroke center. Despite only 3\% of code stroke patients being diagnosed with concomitant COVID-19, we observed longer delays between stroke onset, initial evaluation, and reperfusion therapy. In addition, a lower proportion of acute stroke patients in the pandemic cohort benefited from such therapies. These findings support concerns that the current pandemic may have a negative impact on the acute management of nonCOVID-19-related conditions such as acute stroke. Further research will be needed to investigate the long-term effects of the pandemic on population-based acute stroke incidence, hospital stroke volumes, treatment metrics, and long-term outcomes.

\section{Disclosures}

The authors report no disclosures relevant to the study.

\section{Statement of Authorship}

JNB, CD, and AYP: Conceptualized and designed the study, collected and analyzed the data, and drafted the manuscript. GJ: 
Collected and analyzed the data, and reviewed the manuscript for intellectual content. WA, WB, ND, YD, JE, LCG, DI, CO, JR, $\mathrm{DR}, \mathrm{CS}$, and AW: Reviewed the manuscript for intellectual content.

\section{Supplementary Material}

To view supplementary material for this article, please visit https://doi.org/10.1017/cjn.2020.160.

\section{REFERENCES}

1. Markus HS, Brainin M. COVID-19 and stroke-a global World Stroke Organization perspective. Int J Stroke 2020. doi: 10.1177/ 1747493020923472.

2. Lyden P. Temporary emergency guidance to US stroke centers during COVID-19 pandemic on behalf of the AHA/ASA Stroke Council Leadership. Stroke 2020. doi: 10.1161/STROKEAHA.120.030023.

3. Smith EE, Mountain A, Hill MD, et al. Canadian stroke best practice guidance during the COVID-19 pandemic. Can J Neurol Sci. 2020. doi: $10.1017 /$ cjn.2020.74.
4. Nguyen TN, Abdalkader M, Jovin TG, et al. Mechanical thrombectomy in the era of the COVID-19 pandemic: emergency preparedness for neuroscience teams: a guidance statement from the society of vascular and interventional neurology. Stroke 2020. doi: 10.1161/STROKEAHA.120.030100.

5. Kansagra AP, Goyal MS, Hamilton S, Albers GW. Collateral effect of COVID-19 on stroke evaluation in the United States. N Engl J Med. 2020. doi: 10.1056/NEJMc2014816.

6. Onteddu SR, Nalleballe K, Sharma R, Brown AT. Underutilization of Healthcare for strokes during the COVID-19 outbreak. Int J Stroke 2020:1747493020934362.

7. Pasarikovski CR, da Costa L. The impact of the COVID-19 pandemic on stroke volume. Can J Neurol Sci. 2020. doi: 10.1017/cjn.2020. 116.

8. Rudilosso S, Laredo C, Vera V, et al. Acute stroke care is at risk in the era of COVID-19: experience at a comprehensive stroke center in Barcelona. Stroke 2020. doi: 10.1161/STROKEAHA.120. 030329.

9. Sharma D, Rasmussen M, Han R, et al. Anesthetic management of endovascular treatment of acute ischemic stroke during COVID19 pandemic: consensus statement from Society for Neuroscience in Anesthesiology \& Critical Care (SNACC). J Neurosurg Anesthesiol. 2020. doi: 10.1097/ANA.0000000000000688 